\title{
DYNAMICAL SYSTEMS OF PROBABILISTIC ORIGIN: GAUSSIAN AND POISSON SYSTEMS
}

\author{
ÉLISE JANVRESSE, EMMANUEL ROY AND THIERRY DE LA RUE
}

\section{OutLine}

Glossary

1. Definition of the subject

2. Introduction

3. From probabilistic objects to dynamical systems

3.1. Gaussian systems

3.2. Poisson suspensions

4. Spectral theory

4.1. Basics of spectral theory

4.2. Fock space

4.3. Operators on a Fock space

4.4. Application to Gaussian and Poisson chaos

5. Basic ergodic properties

5.1. Ergodicity and mixing

5.2. Entropy, Bernoulli properties

6. Joinings, factors and centralizer

6.1. Gaussian factors and centralizer

6.2. Poisson factors and centralizer

6.3. Gaussian and Poisson self-joinings

7. GAGs and PAPs

7.1. From Foias-Stratila to GAGs

7.2. Poissonian analog of Foias-Stratila Theorem and PAPs

7.3. Properties of GAGs and PAPs 17

8. Future Directions 17

$\begin{array}{ll}\text { References } & 18\end{array}$

\section{GLOSSARY}

Centralizer. The centralizer of an invertible measure-preserving transformation $T$ is the set $C(T)$ of all invertible measure-preserving transformations on the same measure space which commute with $T$.

(Simple) Counting measure. A counting measure on a measurable space $(X, \mathscr{A})$ is a measure of the form $\sum_{i \in I} \delta_{x_{i}}$ where $\left(x_{i}\right)_{i \in I}$ is a countable family of elements of $X$. The counting measure is said to be simple if $x_{i} \neq x_{j}$ whenever $i \neq j$. 
Gaussian process, Gaussian space. A Gaussian process is a family of real-valued random variables defined on a probability space $(\Omega, \mathbb{P})$, such that any linear combination of finitely many of these random variables is either 0 or normally distributed.

A real linear subspace of $L^{2}(\mathbb{P})$ is a Gaussian space if any nonzero random variable it contains is normally distributed. The closure of the linear real subspace spanned by a Gaussian process is a Gaussian space.

Infinite divisibility. Let $(G, \mathcal{G},+)$ be a measurable Abelian semigroup, i.e. the addition

$$
\begin{aligned}
(G \times G, \mathcal{G} \otimes \mathcal{G}) & \rightarrow(G, \mathcal{G}) \\
\left(g_{1}, g_{2}\right) & \mapsto g_{1}+g_{2}
\end{aligned}
$$

is commutative and measurable. The convolution $\nu * \rho$ of probability measures $\nu$ and $\rho$ on $(G, \mathcal{G})$ is well defined as the image of $\nu \otimes \rho$ by the addition.

A probability measure $\nu$ on $(G, \mathcal{G})$ is infinitely divisible if for any $k \geq 1$, there exists a probability measure $\nu_{k}$ on $(G, \mathcal{G})$ such that

$$
\nu=\left(\nu_{k}\right)^{* k} \text {. }
$$

Kronecker subset of the unidimendional torus. A subset $K$ of the unidimendional torus $\mathbb{T}=\mathbb{R} / \mathbb{Z}$ is a Kronecker set if any continuous function $f: K \rightarrow S^{1}$ is a uniform limit of characters: there exists a sequence $\left(k_{n}\right) \subset \mathbb{Z}$ such that

$$
\max _{t \in K}\left|f(t)-e^{i 2 \pi k_{n} t}\right| \underset{n \rightarrow \infty}{\longrightarrow} 0 \text {. }
$$

Any finite set of rationally independent elements of $\mathbb{T}$ is a Kronecker set, but there exist also perfect Kronecker subsets of $\mathbb{T}$ (see for example [2, Appendix 4]).

Point process. A point process $N$ on $(X, \mathscr{A})$ is a random variable taking values in the space $X^{*}$ of counting measures on $(X, \mathscr{A})$. It is said to be simple if $N$ is almost surely a simple counting measure.

The measure $A \mapsto \mathbb{E}[N(A)]$ on $(X, \mathcal{A})$ is called the intensity of $N$.

A point process of intensity $\mu$ is said to have moment of order $k \geq 1$ if, for all $A \in \mathscr{A}$ with $0<\mu(A)<\infty$,

$$
\mathbb{E}\left[N(A)^{k}\right]<\infty
$$

Poisson point process. Let $(X, \mathscr{A}, \mu)$ be a sigma-finite measure space. A Poisson point process of intensity $\mu$ on $X$ is a point process $N$ on $X$, such that

- For any set $A \in \mathscr{A}$ with $0<\mu(A)<\infty, N(A)$ is a Poisson random variable of parameter $\mu(A)$.

- For any $k \geq 1$, for any collection $\left(A_{1}, \ldots, A_{k}\right)$ in $\mathscr{A}$, the random variables $N\left(A_{i}\right), 1 \leq i \leq k$, are independent.

A Poisson process is simple if and only if its intensity is a continuous measure.

Self-joining and associated Markov operator. A self-joining of the probability-preserving dynamical system $(X, \mathscr{A}, \mu, T)$ is a probability measure $\nu$ on the Cartesian square $(X \times X, \mathscr{A} \otimes \mathscr{A})$ such that

- both marginals of $\nu$ are equal to $\mu$,

- $\nu$ is $T \times T$-invariant. 
When $\nu$ is such a self-joining, it gives rise to a bigger probability-preserving dynamical system $(X \times X, \mathscr{A} \otimes \mathscr{A}, \nu, T \times T)$ in which we see two copies of the original system as factors (via the projections on the two coordinates).

To a self-joining $\nu$ there corresponds a unique Markov operator $\Phi_{\nu}$ (i.e. $\Phi_{\nu}$ is a positive operator on $L^{2}(\mu)$ with $\Phi_{\nu} 1=1$ ) that commutes with the Koopman operator $U_{T}$ (i.e. the unitary operator defined on $L^{2}(\mu)$ by $U_{T} h:=h \circ T$ ), and which is characterized by the following relation: for any $A$ and $B$ in $\mathscr{A}$ :

$$
\nu(A \times B)=\left\langle 1_{A}, \Phi_{\nu} 1_{B}\right\rangle_{L^{2}(\mu)} .
$$

Stationary process. A stationary process is a sequence $\left(\xi_{n}\right)_{n \in \mathbb{Z}}$ of random variables taking values in a set $V$, such that for each $k \in \mathbb{Z}$ and any $i_{1}<\cdots<i_{d} \in \mathbb{Z}$, the distribution of $\left(\xi_{i_{1}+k}, \ldots, \xi_{i_{d}+k}\right)$ is the same as the distribution of $\left(\xi_{i_{1}}, \ldots, \xi_{i_{d}}\right)$. In other words, the process is stationary if its distribution is shift-invariant on $V^{\mathbb{Z}}$.

Spectral measure. Let $U$ be a unitary operator on a Hilbert space $H$. For any $h \in H$, there exists a finite positive measure $\sigma_{h}$ on the unidimendional torus, called the spectral measure of $h$, satisfying for all $k \in \mathbb{Z}$

$$
\widehat{\sigma}_{h}(k):=\int_{\mathbb{T}} e^{-i 2 \pi k t} d \sigma_{h}(t)=\left\langle h, U^{k} h\right\rangle .
$$

When $T$ is an invertible measure-preserving transformation on a measure space $(X, \mathscr{A}, \mu)$, this applies in particular to the associated Koopman operator $U_{T}$. In the case of a square integrable stationary process $\left(\xi_{n}\right)_{n \in \mathbb{Z}}$ (see definition below), its distribution being shift-invariant, the covariances $\mathbb{E}\left[\xi_{0} \overline{\xi_{k}}\right]$ of the process can be interpreted as scalar products $\left\langle h, U^{k} h\right\rangle$, where $h$ is the projection on the 0 coordinate of the process, and $U$ is the Koopman operator associated to the shift. Thus the spectral measure $\sigma$ of the process satisfies, for all $k \in \mathbb{Z}$,

$$
\widehat{\sigma}(k)=\mathbb{E}\left[\xi_{0} \overline{\xi_{k}}\right] .
$$

In the case of a real-valued stationary process, the covariances are real and this measure $\sigma$ must be symmetric (invariant by $t \mapsto-t$ ).

\section{Definition of The SUBJECT}

Measure-theoretic dynamical systems are systems of the form $(X, \mathscr{A}, \mu, T)$ where $(X, \mathscr{A})$ is a standard Borel space, $\mu$ is a sigma-finite measure on $(X, \mathscr{A})$ and $T$ : $X \rightarrow X$ is an invertible measurable transformation preserving $\mu$. In many cases, $\mu$ is a probability measure, and the theory of probability-preserving dynamical systems has considerably developped since the mid 20-th century. In the immense zoo of examples that have caught the interest of mathematicians, very interesting and fundamental families are directly issued from probability theory. The purpose of this article is to present two of them: systems arising from Gaussian processes, and systems constructed from Poisson point processes. Although these two families are of different nature, and each one has to be addressed with specific techniques, a good reason to present them in parallel is that they share striking common features. 


\section{INTRODUCTION}

As we all know, Gaussian distribution plays a prominent role in probability theory and in mathematics in general. It comes with a very rich structure, interesting objects and results, that fueled intensive studies by countless mathematicians. Therefore, it comes as no surprise that Gaussian distribution had its declination in ergodic theory, in the form of stationary Gaussian processes, and received much attention over the years. Their basic ergodic properties started to be studied in the middle of the 20-th century, especially by Ito [14], Maruyama [31] and Fomin [8]. As we can completely control their spectral structure with a relatively simple object which is the spectral measure of the generating process, Gaussian systems have proved to be a very rich source of examples of measure-theoretic dynamical systems with specific properties (see e.g. the examples given by Girsanov [11], Totoki [52] and Newton [36]).

The Poisson point process has a long history. It was first considered on the line to represent the occurrences of independent events over time. The idea of letting the random points move to model the behaviour of a large number of particles $[6,5]$ led to study the Poisson point process on more general spaces. The first formalization of Poisson suspensions, due to Goldstein, Lebowitz and Aizenman in 1975 [12], was also motivated by questions of statistical physics. Simultaneously and independently, Vershik, Gel'fand and Graev [53] considered the action of a group of transformations on configurations of random points. This initiated the systematic study of ergodic properties of these systems, in particular by Marchat [30], Grabinsky [13] and Kalikow [22].

It is worth mentionning Maruyama [33] again who used both Poisson processes and Gaussian stationary processes as a tool to represent any infinitely divisible stationary processes and obtained results on ergodicity and mixing for those processes.

\section{From PRobabilistic OBJECTS TO DYNAMICAL SYSTEMS}

\subsection{Gaussian systems.}

Stationary Gaussian process. We only consider here Gaussian processes $\left(\xi_{n}\right)_{n \in \mathbb{Z}}$ which are centered: $\mathbb{E}\left[\xi_{n}\right]=0$ for each $n$. A fundamental property of Gaussian processes is the fact that their distribution law is completely determined by the covariances $\mathbb{E}\left[\xi_{i} \xi_{j}\right]$. In particular, such a process is stationary if and only if the covariances satisfy, for all $i, j \in \mathbb{Z}, \mathbb{E}\left[\xi_{i} \xi_{j}\right]=\mathbb{E}\left[\xi_{i-j} \xi_{0}\right]$.

In the stationary case, the law of the Gaussian process is therefore completely determined by its spectral measure, which is a symmetric positive finite measure $\sigma$ on $\mathbb{T}$ whose Fourier coefficients are given by (2).

Standard Gaussian systems. Conversely, given a symmetric positive finite measure $\sigma$ on $\mathbb{T}$, there exists a unique (up to equality in distribution) centered stationary Gaussian process whose covariances are given by $(2)^{1}$. The distribution $\mu_{\sigma}$ of this stationary Gaussian process is shift-invariant, thus we can consider the probabilitypreserving dynamical system

$$
\boldsymbol{X}_{\sigma}:=\left(\mathbb{R}^{\mathbb{Z}}, \mathscr{B}\left(\mathbb{R}^{\mathbb{Z}}\right), \mu_{\sigma}, S\right)
$$

where $S$ denotes the shift map: $\left(x_{n}\right)_{n \in \mathbb{Z}} \mapsto\left(x_{n+1}\right)_{n \in \mathbb{Z}}$.

\footnotetext{
${ }^{1}$ In some sense, this ability of realizing any spectral measure characterizes Gaussian processes (see Section 7.1).
} 
A standard Gaussian system is a probability-preserving dynamical system $(X, \mathscr{B}, \mu, T)$ isomorphic to $\boldsymbol{X}_{\sigma}$ for some symmetric positive finite measure $\sigma$ on $\mathbb{T}$. In other words, $(X, \mathscr{B}, \mu, T)$ is a standard Gaussian system if the sigma-algebra $\mathscr{B}$ is generated by some Gaussian process $\left(\xi_{n}\right)_{n \in \mathbb{Z}}$ with $\xi_{n}=\xi_{0} \circ T^{n}$ for each $n \in \mathbb{Z}$. (Multiplying if necessary the process $\left(\xi_{n}\right)$ by a constant, we can always assume that $\mathbb{E}\left[\xi_{0}^{2}\right]=1$, and in this case the spectral measure $\sigma$ is a probability measure.)

The classical theory of Gaussian systems studies the above-mentioned standard Gaussian systems. But following [25], it is useful to introduce also the generalized Gaussian systems as probability-preserving dynamical systems $(X, \mathscr{B}, \mu, T)$ satisfying the following more general property: there exists a closed real subspace $H$ of $L^{2}(\mu)$ such that

- $H$ is a Gaussian space;

- $H$ is invariant by $U_{T}$ (in particular, the stationary process $\left(\xi \circ T^{n}\right)_{n \in \mathbb{Z}}$ is Gaussian);

- the sigma-algebra generated by $H$ is $\mathscr{B}$.

Geometric interpretation. A geometric model for a standard Gaussian dynamical system is proposed in [45] as a transformation of a complex Brownian motion path. More precisely, let $B=\left(B_{t}\right)_{0 \leq t \leq 1}$ be a complex Brownian motion with $B_{0}=0$. For any given probability measure $\gamma$ on $\mathbb{T}$ (identified here with $[0,1[$ ), let us define, for $0 \leq s<1$

$$
\theta(s):=\inf \{x \in[0,1[: \gamma([0, x]) \geq s\} .
$$

Now we consider a new process $\widetilde{B}=\left(\widetilde{B}_{t}\right)_{0 \leq t \leq 1}$ given by

$$
\widetilde{B}_{t}:=\int_{0}^{t} e^{i 2 \pi \theta(s)} d B_{s}
$$

Then $\widetilde{B}$ is also a complex Brownian motion with the same law as $B$, and this enables us to define a transformation $T_{\gamma}: B \mapsto \widetilde{B}$ of the canonical space $C_{0}([0,1], \mathbb{C})$ of the Brownian motion, preserving the Wiener measure $\mu_{W}$. This transformation is invertible (its inverse being given by a similar formula, where we replace $e^{i 2 \pi \theta(s)}$ by $\left.e^{-i 2 \pi \theta(s)}\right)$. Then we define the real valued stationary process $\left(\xi_{p}\right)_{p \in \mathbb{Z}}$ by

$$
\xi_{p}:=\Re \mathrm{e}\left(B_{1} \circ T_{\gamma}^{p}\right) .
$$

It turns out that $\left(\xi_{p}\right)_{p \in \mathbb{Z}}$ is a stationary Gaussian process, whose spectral measure $\sigma$ is the symmetrized of $\gamma$, defined for each measurable subset $A$ of $\mathbb{T}$ by

$$
\sigma(A)=\frac{\gamma(A)+\gamma(-A)}{2} .
$$

Moreover, if $\sigma$ is continuous, $\left(\xi_{p}\right)_{p \in \mathbb{Z}}$ generates the same sigma-algebra as $\left(B_{t}\right)_{0 \leq t \leq 1}$, and the measure-preserving system $\left(C_{0}([0,1], \mathbb{C}), \mu_{W}, T_{\gamma}\right)$ is a Gaussian dynamical system isomorphic to $\boldsymbol{X}_{\sigma}$.

\subsection{Poisson suspensions.}

Poisson point process. Let $(X, \mathscr{A})$ be a standard Borel space, and let $\left(X^{*}, \mathscr{A}^{*}\right)$ be the canonical space of point processes on $(X, \mathscr{A})$, where

- $X^{*}$ is the set of counting measures on $X$, 
- $\mathscr{A}^{*}$ is the sigma-algebra generated by the maps $\left(\mathscr{N}_{A}\right)_{A \in \mathscr{A}}$, where

$$
\mathscr{N}_{A}:\left\{\begin{array}{l}
X^{*} \rightarrow \mathbb{N} \cup\{+\infty\} \\
\omega \mapsto \omega(A) .
\end{array}\right.
$$

When $\mu$ is a sigma-finite measure on $(X, \mathscr{A})$, a Poisson point process of intensity $\mu$ on $X$ always exists. We denote by $\mu^{*}$ its distribution, which is a probability measure on $\left(X^{*}, \mathscr{A}^{*}\right)$.

Poisson suspensions. The distribution $\mu^{*}$ of a Poisson point process of intensity $\mu$ has many nice functorial features. One of the most important is the following.

Let $\mu$ and $\nu$ be two sigma-finite measures on the standard Borel spaces $(X, \mathscr{A})$ and $(Y, \mathscr{B})$ respectively, and consider a measurable map $\varphi: X \rightarrow Y$ such that $\mu \circ \varphi^{-1}=\nu$. Then the map $\varphi_{*} X^{*} \rightarrow Y^{*}$ acting by

$$
\varphi_{*}\left(\sum_{i \in I} \delta_{x_{i}}\right):=\sum_{i \in I} \delta_{\varphi\left(x_{i}\right)}
$$

satisfies $\mu^{*} \circ \varphi_{*}^{-1}=\nu^{*}$.

In particular, if $T$ is a measure-preserving transformation on $(X, \mathscr{A}, \mu)$, then $T_{*}$ is a probability-preserving transformation of $\left(X^{*}, \mathscr{A}^{*}, \mu^{*}\right)$. The system $\left(X^{*}, \mathscr{A}^{*}, \mu^{*}, T_{*}\right)$ is called the Poisson suspension over the base $(X, \mathscr{A}, \mu, T)$.

Of course the properties of the Poisson suspensions depend on those of the base system, and this allows to make strong connections between infinite-measurepreserving systems and probability-preserving systems.

\section{SPECTRAL THEORY}

4.1. Basics of spectral theory. Let us recall some basic notions of spectral theory (see e.g. [27]).

Let $U$ be a unitary operator acting on a separable Hilbert space $H$, and let $h \in H$. We denote by $\sigma_{h}$ the spectral measure of $h$ (see (1)). Let $C(h)$ be the cyclic space of $h$ under $U$, that is, the closure of the linear span of the vectors $U^{n} h$, $n \in \mathbb{Z}$. The linear map between $C(h)$ and $L^{2}\left(\sigma_{h}\right)$ that maps $U^{n} h$ to $e^{i 2 \pi n \cdot}$ extends to an isometry and intertwins the operator $U$ with the unitary operator

$$
V: f \mapsto e^{i 2 \pi \cdot} f, \quad f \in L^{2}\left(\sigma_{h}\right) .
$$

The maximal spectral type of $U$ is the equivalence class of $\sigma_{h_{\max }}$ for some $h_{\max }$ satisfying:

$$
\forall g \in H, \sigma_{g} \ll \sigma_{h_{\max }} .
$$

(Such an $h_{\max }$ always exists.)

We say that $U$ has simple spectrum whenever $H$ itself is a cyclic space $C(h)$ for some vector $h$.

Whenever $(X, \mathscr{A}, \mu, T)$ is a measure-preserving dynamical system with a sigmafinite measure $\mu$, we denote by $U_{T}: L^{2}(\mu) \rightarrow L^{2}(\mu)$ the associated Koopman operator defined by $U_{T} h:=h \circ T$. It is a unitary operator on $L^{2}(\mu)$. 
4.2. Fock space. We describe here an algebraic construction that plays a crucial role in the study of our objects.

Let once again $H$ be a Hilbert space and denote $H^{\odot n}$, the vector space of symmetric elements of the $n$-th tensor product $H^{\otimes n}$. When $H$ is $L^{2}(X, \mu)$ for some sigma-finite measure $\mu$ on $X$, then $H^{\odot n}$ can be identified with the subspace $L_{\text {perm }}^{2}\left(X^{n}, \mu^{\otimes n}\right)$ of $L^{2}\left(X^{n}, \mu^{\otimes n}\right)$ consisting of functions which are invariant by coordinate permutations.

With the convention $H^{\odot 0}:=\mathbb{C}$, we can consider the vector space $\sum_{n>0} H^{\odot n}$ which is the formal direct sum whose elements are finite sums of vectors of $H^{\odot n}$, $n \geq 1$. The space $\sum_{n \geq 0} H^{\odot n}$ can be equipped with a scalar product by considering the direct sum as orthogonal and by endowing each $H^{\odot n}$ with the scalar product $\langle\cdot, \cdot\rangle_{H \otimes n}$. The (Boson) Fock space $F(H)$ of $H$ is the Hilbert space obtained as the completion of $\sum_{n \geq 0} H^{\odot n}$ with respect to the norm of the scalar product we just set up.

4.3. Operators on a Fock space. Whenever $\Phi$ is an operator on $H$ of norm less than or equal to 1 , it extends naturally to an operator $\widetilde{\Phi}$ on the Fock space $F(H)$ by acting on $H^{\odot n}$ as $\Phi^{\odot n}$, that is

$$
\forall v \in H, \quad \widetilde{\Phi}(v \otimes \cdots \otimes v):=\Phi v \otimes \cdots \otimes \Phi v .
$$

This operator $\widetilde{\Phi}$ is called the second quantization of $\Phi$ (see [1] (chap. 8, p. 16)).

The following proposition considers the second quantization $\widetilde{U}$ of a unitary operator $U$.

Proposition 4.1. If $U$ is unitary on $H$ and $\sigma_{\max }$ is in the equivalence class of its maximal spectral type, then

- the second quantization $\widetilde{U}$ of $U$ is unitary on $F(H)$.

- The maximal spectral type of $\widetilde{U}$ is the equivalence class of $\sum_{n \geq 0} \sigma_{\max }^{* n}$ (where $\left.\sigma_{\max }^{* 0}:=\delta_{0}\right)$.

- $\widetilde{U}$ has simple spectrum if and only if for all $n, U^{\odot n}$ has simple spectrum and for all $n \neq m, \sigma_{\max }^{* n} \perp \sigma_{\max }^{* m}$.

\subsection{Application to Gaussian and Poisson chaos.}

4.4.1. Fock space structure of $L^{2}$ for Gaussian dynamical systems and Poisson suspensions. In the case of the standard Gaussian dynamical system $\boldsymbol{X}_{\sigma}$, generated by the Gaussian process $\left(\xi_{n}\right)_{n \in \mathbb{Z}}$, we denote by $H_{1}^{r}$ the real Gaussian subspace of $L^{2}\left(\mu_{\sigma}\right)$ spanned by the random variables $\xi_{n}, n \in \mathbb{Z}$, and $H_{1}:=H_{1}^{r}+i H_{1}^{r}$ the complex subspace spanned by the process. Then $H_{1}$ is isometric to $L^{2}(\mathbb{T}, \sigma)$, with an isometry extending the correspondence $\xi_{n} \longleftrightarrow e^{i 2 \pi n \cdot}(n \in \mathbb{Z})$.

In the case of the Poisson suspension $\left(X^{*}, \mathscr{A}^{*}, \mu^{*}, T_{*}\right)$, we denote by $H_{1}$ the subspace of $L^{2}\left(\mu^{*}\right)$ spanned by the random variables of the form $\mathscr{N}_{A}-\mu(A), A \in \mathscr{A}$, $\mu(A)<\infty$. In this case $H_{1}$ is isometric to $L^{2}(X, \mu)$, with an isometry extending the correspondence $\mathscr{N}_{A}-\mu(A) \longleftrightarrow 1_{A}(A \in \mathscr{A}, \mu(A)<\infty)$.

In both cases, $H_{0}$ denotes the subspace of constant functions. Then for each $n \geq$ 2 , we define inductively the subspace $H_{n}$ as the orthocomplement of $\bigoplus_{0 \leq j<n} H_{j}$ in the space of all polynomials of degree at most $n$ in variables in $H_{1}$ (note that elements of $H_{1}$ always have moments of any order). In the classical probabilist terminology, the subspace $H_{n}$ is called the $n$-th chaos. 
It turns out that $H_{n}$ is, in the Gaussian case, isometric to $L_{\text {perm }}^{2}\left(\mathbb{T}^{n}, \sigma^{\otimes n}\right)$, whereas in the Poisson case it is isometric to $L_{\text {perm }}^{2}\left(X^{n}, \mu^{\otimes n}\right)$. We therefore get the following description of $L^{2}$ as a Fock space, which in the Gaussian case takes the form

$$
L^{2}\left(\mu_{\sigma}\right)=\bigoplus_{n \geq 0} H_{n} \simeq F\left(L^{2}(\mathbb{T}, \sigma)\right)
$$

and in the Poisson case

$$
L^{2}\left(\mu^{*}\right)=\bigoplus_{n \geq 0} H_{n} \simeq F\left(L^{2}(X, \mu)\right)
$$

We refer to [2] for more details about the Fock space structure in the Gaussian case, and to $[24,35]$ in the Poisson case.

4.4.2. Second quantization operators. Once the Fock space structure of $L^{2}\left(\mu_{\sigma}\right)$ (in the Gaussian case) and $L^{2}\left(\mu^{*}\right)$ (in the Poisson suspension case) is established, it is natural to look at the Koopman operator that is associated to the underlying transformation. In both cases, this Koopman operator leaves each chaos $H_{n}$ invariant, and is nothing but the second quantization of its restriction to $H_{1}$. More precisely, we can state the following.

In the case of the standard Gaussian system $\boldsymbol{X}_{\sigma}$, the action of $U_{S}$ on the first chaos $H_{1}$ corresponds to the multiplication by $e^{i 2 \pi \cdot}$ in $L^{2}(\mathbb{T}, \sigma)$. In general, the action of $U_{S}$ on the $n$-th chaos $H_{n}$ corresponds to the multiplication by the function $\left(t_{1}, \ldots, t_{n}\right) \mapsto e^{i 2 \pi\left(t_{1}+\cdots+t_{n}\right)}$ in $L_{\text {perm }}^{2}\left(\mathbb{T}^{n}, \sigma^{\otimes n}\right)$.

In the case of the Poisson suspension $\left(X^{*}, \mathscr{A}^{*}, \mu^{*}, T_{*}\right)$, the action of $U_{T_{*}}$ on $H_{1}$ corresponds to the action of $U_{T}$ on $L^{2}(\mu)$. In general, the action of $U_{T_{*}}$ on the $n$-th chaos $H_{n}$ corresponds to the action of $U_{T \times n}$ on $L_{\text {perm }}^{2}\left(X^{n}, \mu^{\otimes n}\right)$.

\section{BASIC ERGODIC PROPERTIES}

\subsection{Ergodicity and mixing.}

Ergodic properties as spectral properties. We list some classical ergodic properties that are spectral by nature. We start by the probability-preserving case where it is customary to consider $U_{T}: f \mapsto f \circ T$ acting on $L_{0}^{2}(\mu)=L^{2}(\mu) \ominus \mathbb{C}$, the orthocomplement of the constant functions as $U_{T}$ acts trivially on the latter: $U_{T}\left(\alpha 1_{X}\right)=\alpha 1_{X}$. In that case, the reduced maximal spectral type denotes the maximal spectral type of $U_{T}$ acting on $L_{0}^{2}(\mu)$.

Theorem 5.1. Let $(X, \mathscr{A}, \mu, T)$ be a dynamical system with $\mu(X)=1, U_{T}$ its Koopman operator acting on $L_{0}^{2}(\mu)$ and $\rho_{\text {max }}^{0}$, a finite measure in the measure class of the reduced maximal spectral type.

- $(X, \mathscr{A}, \mu, T)$ is ergodic if and only if $\rho_{\max }^{0}(\{0\})=0$.

- $(X, \mathscr{A}, \mu, T)$ is weakly mixing if and only if $\rho_{\max }^{0}$ is continuous.

- $(X, \mathscr{A}, \mu, T)$ is (strongly) mixing if and only if $\rho_{\max }^{0}$ is Rajchman, i.e. $\widehat{\rho_{\max }^{0}}(k) \underset{|k| \rightarrow \infty}{\longrightarrow} 0$.

When $\mu$ is not finite, nonzero constant functions are not in $L^{2}(\mu)$ and that makes a radical difference: 
Theorem 5.2. Let $(X, \mathscr{A}, \mu, T)$ be a dynamical system with $\mu$ sigma-finite, $U_{T}$ its Koopman operator acting on $L^{2}(\mu)$ and $\rho_{\max }$ a finite measure in the measure class of the maximal spectral type.

- $(X, \mathscr{A}, \mu, T)$ has no T-invariant set of finite positive measure if and only if $\rho_{\max }$ is continuous.

- $(X, \mathscr{A}, \mu, T)$ is of zero type (i.e. $\mu\left(A \cap T^{n} B\right) \rightarrow 0$ as $n$ tends to infinity for all finite $\mu$-measure sets $A$ and $B$ in $\mathscr{A}$ ) if and only if $\rho_{\max }$ is Rajchman.

In particular, these two properties can only happen if $\mu$ is infinite. Note also that ergodicity is no longer a spectral property in the infinite measure case.

Ergodicity and mixing of Gaussian systems and Poisson suspensions. Ergodic and mixing properties of Gaussian dynamical systems are easily characterized in terms of the spectral measure $\sigma$ of the generating stationary Gaussian process. Using the spectral characterizations of ergodicity and mixing given in Theorem 5.1 and the expression of the maximal spectral type of the second quantization of a unitary operator in Proposition 4.1, we can obtain the following results which were first proved by Maruyama [31] and Fomin [8]. Details can also be found in [2].

Theorem 5.3. The Gaussian dynamical system $\boldsymbol{X}_{\sigma}$ is ergodic if and only if the spectral measure $\sigma$ is continuous, that is $\sigma(\{t\})=0$ for each $t \in \mathbb{T}$. In this case it is also weakly mixing.

The system $\boldsymbol{X}_{\sigma}$ is (strongly) mixing if and only if $\sigma$ is Rajchman.

In particular, by the Riemann-Lebesgue Lemma, if $\sigma$ is absolutely continuous then the associated Gaussian system is mixing, which was originately proved by Ito [14]. But there also exist singular spectral measures whose Fourier coefficients vanish at infinity [34].

The same kind of arguments apply to Poisson suspensions: together with Theorem 5.2, this yields to the following characterizations (proved by Marchat [30] and Grabinsky [13]).

Theorem 5.4. The Poisson suspension $\left(X^{*}, \mathscr{A}^{*}, \mu^{*}, T_{*}\right)$ is ergodic if and only if the base system $(X, \mathscr{A}, \mu, T)$ has no invariant set of finite positive measure. In this case it is also weakly mixing.

The Poisson suspension $\left(X^{*}, \mathscr{A}^{*}, \mu^{*}, T_{*}\right)$ is (strongly) mixing if and only if the base system $(X, \mathscr{A}, \mu, T)$ is of zero type.

Leonov [29] proved that, if a Gaussian system is mixing, then it is mixing of all orders (see also [52] for a simple proof by Totoki). The same result holds for a Poisson suspension (see $[30,13]$ ).

5.2. Entropy, Bernoulli properties. The Kolmogorov-Sinai entropy of a Gaussian system is given by the following result, already stated by Pinsker in 1960 [41] (see also $[37,44,26]$ for various proofs).

Theorem 5.5 (Entropy of a standard Gaussian system). Let $\sigma$ be a symmetric positive finite measure on $\mathbb{T}$. If $\sigma$ is singular with respect to the Lebesgue measure, then $h\left(\boldsymbol{X}_{\sigma}\right)=0$. Otherwise, $h\left(\boldsymbol{X}_{\sigma}\right)=\infty$.

In the case where its spectral measure is the Lebesgue measure $\lambda$ on $\mathbb{T}$, the stationary Gaussian process $\left(\xi_{n}\right)_{n \in \mathbb{Z}}$ is composed of orthogonal, hence independent, random variables. Thus $\boldsymbol{X}_{\lambda}$ is a Bernoulli shift of infinite entropy. Now if $\sigma \ll \lambda$, 
we will see in Theorem 6.1 that $\boldsymbol{X}_{\sigma}$ is a factor of a Bernoulli shift. By Ornstein theory [39], $\boldsymbol{X}_{\sigma}$ is itself isomorphic to a Bernoulli shift, and since it also has infinite entropy by Theorem 5.5 , it is in fact isomorphic to $\boldsymbol{X}_{\lambda}$.

In the general case, decomposing the spectral measure as the sum of its singular and absolutely continuous parts and assuming that both are nonzero, we get by Theorem 6.1 below that $\boldsymbol{X}_{\sigma}$ is the direct product of a Bernoulli shift of infinite entropy with a zero-entropy Gaussian system (corresponding to the singular part of the spectral measure). In particular, any standard Gaussian system satisfies the Pinsker property (it is a product of a zero-entropy system with a Bernoulli shift).

As far as Poisson suspensions are concerned, anything can happen with the entropy which can be 0 , positive finite or infinite. Together with the fact that, when the base $(X, \mathscr{A}, \mu, T)$ is a probability space, the entropy of the Poisson suspension $T_{*}$ is just the same as the entropy of $T$, this leads to view the entropy of the Poisson suspension $T_{*}$ as a possible way to define the entropy of $T$ when $T$ is an infinite measure-preserving transformation, as proposed in [43]. Other ways to define the entropy of an infinite measure-preserving transformation, also generalizing the finite-measure case, had been proposed by Krengel [23] and Parry [40], and it is proved in [18] that these three notions of entropy coincide in many cases. However an example of a transformation with zero Krengel entropy, but whose Poisson suspension has positive entropy, is presented in [17].

Assume that there exists in the base system $(X, \mathscr{A}, \mu, T)$ a wandering set $A$ (i.e. whose images $T^{n} A, n \in \mathbb{Z}$, are pairwise disjoint), and such that $X=\bigcup_{n \in \mathbb{Z}} T^{n} A$ (the system is called dissipative in this case). Then, obviously, the base is of zero type and the Poisson suspension is mixing. But thanks to the independence property of the Poisson process, the Poisson suspension is in fact Bernoulli. However there also exist Bernoulli examples of Poisson suspensions where the base is conservative (i.e. there is no wandering set of positive measure). Such examples are provided by Poisson suspensions over null-recurrent Markov chains: we consider a countable set $\Sigma$, an irreducible null-recurrent stochastic matrix $P=\left(p_{i, j}\right)_{i, j \in \Sigma}$, and let $q=\left(q_{i}\right)_{i \in \Sigma}$ be a nonzero measure which is stationary with respect to $P$. We can then form the associated Markov shift $(X, \mathscr{A}, \mu, T)$ where $X=\Sigma^{\mathbb{Z}}, T$ is the shift transformation, and $\mu$ is the shift invariant infinite measure given on cylinder sets by the formula

$$
\mu\left(\left[i_{0} i_{1}, \ldots, i_{k}\right]\right):=q_{i_{0}} p_{i_{0}, i_{1}} \cdots p_{i_{k-1}, i_{k}} .
$$

It has been proved by Kalikow [22] and Grabinsky [13] that the associated Poisson suspension $\left(X^{*}, \mathscr{A}^{*}, \mu^{*}, T_{*}\right)$ is Bernoulli. Moreover, as shown in [18], its entropy is given by the following formula generalizing the entropy for positive-recurrent Markov chains:

$$
h\left(T_{*}\right)=-\sum_{i \in \Sigma} q_{i} \sum_{j \in \Sigma} p_{i, j} \log p_{i, j} .
$$

(Examples where the above entropy is finite are provided by Krengel [23].)

The general structure of a Poisson suspension is, in general, not known. We can ask in particular whether there exists a Poisson suspension which is K but not Bernoulli.

\section{JOININGS, FACTORS AND CENTRALIZER}

\subsection{Gaussian factors and centralizer.}


Gaussian factors of a Gaussian system. In the standard Gaussian system $\boldsymbol{X}_{\sigma}$ generated by the Gaussian process $\left(\xi_{n}\right)_{n \in \mathbb{Z}}$, let us take a nonzero element $\zeta \in H_{1}^{r}$, and set for each $n \in \mathbb{Z}, \zeta_{n}:=\zeta \circ S^{n} \in H_{1}^{r}$. Then $\left(\zeta_{n}\right)_{n \in \mathbb{Z}}$ is a stationary Gaussian process, and generates a particular factor sigma-algebra of $\boldsymbol{X}_{\sigma}$ which we call a Gaussian factor. This factor can be more precisely described by the following analysis.

Spectral theory provides an isometry between the closed real subspace $H_{1}^{r}$ of $L^{2}\left(\mu_{\sigma}\right)$ generated by the Gaussian process $\left(\xi_{n}\right)$ and the following real subspace of $L^{2}(\mathbb{T}, \sigma)$ :

$$
L_{\mathrm{sym}}^{2}(\mathbb{T}, \sigma):=\left\{\phi \in L^{2}(\mathbb{T}, \sigma): \phi(-t)=\overline{\phi(t)} \text { for } \sigma \text {-almost every } t\right\} .
$$

The element $\zeta$ of $H_{1}^{r}$ corresponds to some function $\phi \in L_{\mathrm{sym}}^{2}(\mathbb{T}, \sigma)$, and the spectral measure $\sigma_{1}$ of the process $\left(\zeta_{n}\right)_{n \in \mathbb{Z}}$ is absolutely continuous with respect to $\sigma$, with density $\frac{d \sigma_{1}}{d \sigma}=|\phi|^{2}$.

Observe conversely that any symmetric positive finite measure $\sigma_{1} \ll \sigma$ can be realized in this way, for example by taking the real-valued function $\phi:=\sqrt{\frac{d \sigma_{1}}{d \sigma}} \in$ $L_{\text {sym }}^{2}(\mathbb{T}, \sigma)$.

The Gaussian factor generated by $\left(\zeta_{n}\right)_{n \in \mathbb{Z}}$ only depends on the equivalence class of $\sigma_{1}$, so we can denote it by $\mathscr{F}_{\sigma_{1}}$. The action of $S$ on $\mathscr{F}_{\sigma_{1}}$ is isomorphic to $\boldsymbol{X}_{\sigma_{1}}$.

If $\sigma_{1}$ is equivalent to $\sigma$, then $\mathscr{F}_{\sigma_{1}}$ coincides with the Borel sigma-algebra of $\boldsymbol{X}_{\sigma}$, and $\boldsymbol{X}_{\sigma_{1}}$ is isomorphic to $\boldsymbol{X}_{\sigma}$. On the other hand, if $\sigma_{1}$ is not equivalent to $\sigma$, we can find another symmetric positive finite measure $\sigma_{2}$ on $\mathbb{T}$ such that

- $\sigma_{2}$ is singular with respect to $\sigma_{1}$,

- $\sigma$ is equivalent to $\sigma_{1}+\sigma_{2}$.

Using the fact that orthogonal subspaces of $H_{1}^{r}$ correspond to independent families of Gaussian random variables, we then get another Gaussian factor $\mathscr{F}_{\sigma_{2}}$ which is independent of $\mathscr{F}_{\sigma_{1}}$, and such that $\mathscr{F}_{\sigma_{1}}$ and $\mathscr{F}_{\sigma_{2}}$ together generate the same sigma-algebra as the original Gaussian process $\left(\xi_{n}\right)$. This shows that any strict Gaussian factor $\mathscr{F}_{\sigma_{1}}$ of $\boldsymbol{X}_{\sigma}$ admits an independent complement. In the case when $\boldsymbol{X}_{\sigma}$ is ergodic, this independent complement is itself weakly mixing, therefore the corresponding extension $\boldsymbol{X}_{\sigma} \rightarrow \boldsymbol{X}_{\sigma_{1}}$ is relatively weakly mixing.

We can summarize some of the above results through the following theorem.

Theorem 6.1. Let $\sigma, \sigma_{1}, \sigma_{2}$ be symmetric positive finite measures on $\mathbb{T}$.

- If $\sigma_{1}$ and $\sigma_{2}$ are equivalent, then $\boldsymbol{X}_{\sigma_{1}}$ and $\boldsymbol{X}_{\sigma_{2}}$ are isomorphic.

- If $\sigma_{1} \ll \sigma$, then $\boldsymbol{X}_{\sigma_{1}}$ is a factor of $\boldsymbol{X}_{\sigma}$ and corresponds to a Gaussian factor $\mathscr{F}_{\sigma_{1}}$ of $\boldsymbol{X}_{\sigma}$.

- If $\sigma$ is equivalent to $\sigma_{1}+\sigma_{2}$, where $\sigma_{1}$ and $\sigma_{2}$ are mutually singular, then $\boldsymbol{X}_{\sigma}$ is isomorphic to the direct product $\boldsymbol{X}_{\sigma_{1}} \times \boldsymbol{X}_{\sigma_{2}}$.

Gaussian centralizer. The geometric interpretation of a standard Gaussian dynamical system as a transformation of the Brownian motion path given in Section 3.1 allows to show that the centralizer of a Gaussian system is always rich. First, if in Formula (3) we replace $e^{i 2 \pi \theta(s)}$ by $e^{i 2 \pi u \theta(s)}$ for $u \in \mathbb{R}$, we get a measure-preserving $\mathbb{R}$-action $\left(T_{\gamma}^{u}\right)_{u \in \mathbb{R}}$. Hence any standard Gaussian dynamical system can be embedded in an $\mathbb{R}$-action. Moreover each $T_{\gamma}^{u}$ gives rise to a generalized Gaussian system (as the Gaussian subspace $H_{1}^{r}$ is stable by $T_{\gamma}^{u}$ ). 
Observe also that if we take two probability measures $\gamma_{1}$ and $\gamma_{2}$ on $\mathbb{T}$, then $T_{\gamma_{1}}$ and $T_{\gamma_{2}}$ always commute. Hence the centralizer of a Gaussian dynamical system always contains transformations isomorphic to any other standard Gaussian dynamical system.

The above examples all belong to the so-called Gaussian centralizer, whose elements are constructed as follows. We start by defining the multiplicative group

$$
\left.G_{\sigma}:=\left\{\psi \in L_{\text {sym }}^{2}(\mathbb{T}, \sigma):|\psi|=1 \text { ( } \sigma \text {-a.e. }\right)\right\} .
$$

For each $\psi$ in $G_{\sigma}$, the multiplication by $\psi$ in $L_{\text {sym }}^{2}(\mathbb{T}, \sigma)$ is a unitary operator, which corresponds in $H_{1}^{r}$ to a unitary operator $U_{\psi}$. Moreover, the process $\left(U_{\psi}\left(\xi_{n}\right)\right)_{n \in \mathbb{Z}}$ has the same distribution as the generating Gaussian process $\left(\xi_{n}\right)_{n \in \mathbb{Z}}$, hence $U_{\psi}$ comes from a measure-preserving transformation, which we denote here by $T_{\psi}$, commuting with $S$ on $\boldsymbol{X}_{\sigma}$.

Note that $U_{\psi}$ can be naturally extended to a unitary operator on the complex space $H^{1}$. The Koopman operator associated to $T_{\psi}$ is then nothing but the second quantization of $U_{\psi}$.

The set of transformations

$$
C^{g}(S):=\left\{T_{\psi}: \psi \in G_{\sigma}\right\}
$$

is a subgroup of the centralizer of $S$ which is called the Gaussian centralizer.

Compact and classical factors of a Gaussian dynamical system. Newton and Parry [38] (see also Maruyama [32]) introduced another type of factor of the Gaussian system $\boldsymbol{X}_{\sigma}$ by considering the sigma-algebra of subsets of $\mathbb{R}^{\mathbb{Z}}$ which are invariant by the transformation $\left(x_{n}\right)_{n \in \mathbb{Z}} \mapsto\left(-x_{n}\right)_{n \in \mathbb{Z}}$ (called the even factor). We can see that this factor is not a Gaussian factor, as the corresponding extension is never relatively weakly mixing.

This example belongs to another class of factors of $\boldsymbol{X}_{\sigma}$, which are obtained in the following way. Take any compact subgroup $K$ of $G_{\sigma}$, which corresponds to some compact subgroup $C_{K}$ of $C^{g}(S)$, and consider

$$
\mathscr{F}_{K}:=\left\{A \subset \mathbb{R}^{\mathbb{Z}}: T A=A \text { for each } T \in C_{K}\right\} \text {. }
$$

Then $\mathscr{F}_{K}$ is a factor sigma-algebra of $\boldsymbol{X}_{\sigma}$ which is called a Gaussian-compact factor of $\boldsymbol{X}_{\sigma}$. Note that Newton-Parry's example corresponds to the subgroup $K$ generated by the function identically equal to -1 on $\mathbb{T}$.

Finally, these two types of factors can be combined, and we call a classical factor of $\boldsymbol{X}_{\sigma}$ any Gaussian-compact factor of a Gaussian factor of $\boldsymbol{X}_{\sigma}$.

6.2. Poisson factors and centralizer. There are two main ways to get natural factors of a Poisson suspension of base $(X, \mathscr{A}, \mu, T)$ which are themselves Poisson suspensions. The first one is to consider a non-trivial $T$-invariant set $Y \subset X$ and the sigma-algebra generated by restriction of the Poisson process to $Y$ :

$$
\mathscr{A}_{\mid Y}^{*}:=\sigma\left\{\mathscr{N}_{A}, A \in \mathscr{A}, A \subset Y\right\} .
$$

The associated factor map is $\omega \longmapsto \omega_{\mid Y}$ and sends $\left(X^{*}, \mathscr{A}^{*}, \mu^{*}, T_{*}\right)$ to

$$
\left(Y^{*},\left(\mathscr{A}_{\mid Y}\right)^{*},\left(\mu_{\mid Y}\right)^{*},\left(T_{\mid Y}\right)^{*}\right) \text {. }
$$

As an immediate consequence of the independence properties of a Poisson process, we get the following result. 
Proposition 6.2. Assume there exists a non trivial $T$-invariant set $Y \subset X$. Then the Poisson suspension $\left(X^{*}, \mathscr{A}^{*}, \mu^{*}, T_{*}\right)$ is isomorphic to the direct product

$$
\left(Y^{*} \times(X \backslash Y)^{*},\left(\mathscr{A}_{\mid Y}\right)^{*} \otimes\left(\mathscr{A}_{\mid X \backslash Y}\right)^{*},\left(\mu_{\mid Y}\right)^{*} \otimes\left(\mu_{\mid X \backslash Y}\right)^{*},\left(T_{\mid Y}\right)^{*} \times\left(T_{\mid X \backslash Y}\right)^{*}\right) .
$$

The other way to get a Poisson suspension as a factor of $\left(X^{*}, \mathscr{A}^{*}, \mu^{*}, T_{*}\right)$ is to consider a sigma-finite factor of the base system $\mathscr{C} \subset \mathscr{A}$, that is, a $T$-invariant sigma-algebra where $\mu$ is still sigma-finite. We then define

$$
\mathscr{C}^{*}:=\sigma\left\{\mathscr{N}_{A}, A \in \mathscr{C}\right\} \text {. }
$$

We can check that the action of $T_{*}$ on $\mathscr{C}^{*}$ can be viewed as a Poisson suspension. Indeed, we have the isomorphism

$$
\left(\left(X_{/ \mathscr{C}}\right)^{*},\left(\mathscr{A}_{/ \mathscr{C}}\right)^{*},\left(\mu_{/ \mathscr{C}}\right)^{*},\left(T_{/ \mathscr{C}}\right)^{*}\right) \simeq\left(X_{/ \mathscr{C}^{*}}^{*}, \mathscr{A}_{/ \mathscr{C}}^{*}, \mu_{/ \mathscr{C}}^{*}, T_{* / \mathscr{C}}\right) .
$$

In general, we call Poisson factor of the suspension $\left(X^{*}, \mathscr{A}^{*}, \mu^{*}, T_{*}\right)$ any $T_{*^{-}}$ invariant sub-sigma-algebra of the form $\left(\left.\mathscr{C}\right|_{Y}\right)^{*}$, where $\mathscr{C}$ is a sigma-finite factor of the base system and $Y$ a $\mathscr{C}$-measurable $T$-invariant subset of $X$.

The centralizer of $T_{*}$ always contains other Poisson suspensions, namely all transformations of the form $S_{*}$ when $S \in C(T)$. They form a subgroupg of $C\left(T_{*}\right)$ : the Poisson centralizer.

6.3. Gaussian and Poisson self-joinings. This section is based on notions borrowed from [25] (for Gaussian) and [42], [4] (for Poisson). The inherent probabilistic nature of Gaussian and Poisson systems allows to define remarkable families of selfjoinings.

In the Gaussian setting, the definition is pretty straightforward.

Definition 6.3. Let $\nu$ be a self-joining of a standard Gaussian system $\boldsymbol{X}_{\sigma}$. In the probability-preserving dynamical system

$$
\left(\mathbb{R}^{\mathbb{Z}} \times \mathbb{R}^{\mathbb{Z}}, \mathscr{B}\left(\mathbb{R}^{\mathbb{Z}}\right) \otimes \mathscr{B}\left(\mathbb{R}^{\mathbb{Z}}\right), \nu, S \times S\right),
$$

the two natural projections on $\mathbb{R}^{\mathbb{Z}}$ provide two copies

$$
\left(\xi_{n}^{\prime}\right)=\left(\xi_{0}^{\prime} \circ(S \times S)^{n}\right)_{n \in \mathbb{Z}} \quad \text { and } \quad\left(\xi_{n}^{\prime \prime}\right)=\left(\xi_{0}^{\prime \prime} \circ(S \times S)^{n}\right)_{n \in \mathbb{Z}}
$$

of the original Gaussian process. The self-joining $\nu$ is said to be a Gaussian selfjoining if the real subspace of $L^{2}(\nu)$ spanned by the $\xi_{n}^{\prime}$ and the $\xi_{n}^{\prime \prime}, n \in \mathbb{Z}$, is a Gaussian space.

Example 6.4. The product self-joining, and more generally the relatively independent product over a Gaussian factor, are Gaussian self-joinings. The graph selfjoining associated to a transformation in the Gaussian centralizer is also a Gaussian self-joining. The ergodic components of the relatively independent product over a compact factor are Gaussian self-joinings.

For the Poisson counterpart, the aforementioned family of self-joinings is easier to introduce by expliciting their structure.

Let $\left(X^{*}, \mathscr{A}^{*}, \mu^{*}, T_{*}\right)$ be a Poisson suspension. Start with a "sub-self-joining" of the base, namely a system $(X \times X, \mathscr{A} \otimes \mathscr{A}, m, T \times T)$ where $m$ is a $T \times T$-invariant measure whose projections satisfy

$$
m_{1}:=m(\cdot \times X) \leq \mu \text { and } m_{2}:=m(X \times \cdot) \leq \mu .
$$


We form the Poisson suspension $\left((X \times X)^{*},(\mathscr{A} \otimes \mathscr{A})^{*}, m^{*},(T \times T)_{*}\right)$. Consider the map $\kappa \mapsto\left(\omega_{1}, \omega_{2}\right):=(\kappa(\cdot \times X), \kappa(X \times \cdot))$, and denote by $\bar{m}$ the pushforward measure of $m^{*}$ by this map. Then we get as a factor

$$
\left(X^{*} \times X^{*}, \mathscr{A}^{*} \otimes \mathscr{A}^{*}, \bar{m}, T_{*} \times T_{*}\right),
$$

which is nothing else than a joining between $\left(X^{*}, \mathscr{A}^{*}, m_{1}^{*}, T_{*}\right)$ and $\left(X^{*}, \mathscr{A}^{*}, m_{2}^{*}, T_{*}\right)$.

The final step consists into "fixing the intensities" by considering the direct product

$$
\begin{aligned}
\left(X^{*} \times\left(X^{*} \times X^{*}\right) \times X^{*}, \mathscr{A}^{*} \otimes\right. & \left(\mathscr{A}^{*} \otimes \mathscr{A}^{*}\right) \otimes \mathscr{A}^{*}, \\
& \left.\left(\mu-m_{1}\right)^{*} \otimes \bar{m} \otimes\left(\mu-m_{2}\right)^{*}, T_{*} \times\left(T_{*} \times T_{*}\right) \times T_{*}\right) .
\end{aligned}
$$

On $X^{*} \times X^{*}$, we define $\widetilde{m}$ as the pushforward measure of $\left(\mu-m_{1}\right)^{*} \otimes \bar{m} \otimes\left(\mu-m_{2}\right)^{*}$ by the factor map

$$
\left(\omega^{\prime},\left(\omega_{1}, \omega_{2}\right), \omega^{\prime \prime}\right) \mapsto\left(\omega^{\prime}+\omega_{1}, \omega_{2}+\omega^{\prime \prime}\right) .
$$

We get a self-joining $\left(X^{*} \times X^{*}, \mathscr{A}^{*} \otimes \mathscr{A}^{*}, \widetilde{m}, T_{*} \times T_{*}\right)$ of the original Poisson suspension.

Definition 6.5. We call Poisson self-joining of a Poisson suspension any selfjoining which is obtained as explained above.

Example 6.6. The product self-joining corresponds to the case where $m$ is the null measure. If $S$ is in the centralizer of $T$ and $m:=\Delta_{S}$ is the corresponding graph measure, then $\widetilde{m}=\Delta_{S_{*}}$, i.e. the graph self-joining associated to $S_{*}$. If $\mathscr{C} \subset \mathscr{A}$ is a sigma-finite factor and $m:=\mu \otimes_{\mathscr{C}} \mu$ is the relatively independent joining over $\mathscr{C}$, then $\widetilde{m}=\mu^{*} \otimes_{\mathscr{C}} * \mu^{*}$, i.e. the relatively independent joining over the Poisson factor $\mathscr{C}^{*}$.

Despite the different nature of their structure, it is possible to give a unified characterization of Poisson and Gaussian self-joinings (see [43]).

Proposition 6.7. A self-joining of a Gaussian system (resp. a Poisson suspension) is Gaussian (resp. Poisson) if and only if its distribution is infinitely divisible.

In the Gaussian case, this follows immediately from the fact that a process with Gaussian entries is Gaussian if and only if it is infinitely divisible.

Theorem 6.8. [25, 4] A self-joining of a Gaussian system $\boldsymbol{X}_{\sigma}$ is Gaussian if and only if the associated Markov operator $\Phi$ acting on $L^{2}\left(\mu_{\sigma}\right)$ is the second quantization of an operator $\varphi$ on $L^{2}(\sigma)$ of norm less than or equal to one and which commutes with $V$ (see (4)).

Similarly, a self-joining of a Poisson suspension $\left(X^{*}, \mathscr{A}^{*}, \mu^{*}, T_{*}\right)$ is Poisson if and only if the associated Markov operator $\Phi$ acting on $L^{2}\left(\mu^{*}\right)$ is the second quantization of an operator $\varphi$ on $L^{2}(\mu)$ corresponding to a sub-self-joining of $(X, \mathscr{A}, \mu, T)$ (a sub-Markov operator commuting with $T$ ).

The following property is central in this theory (see [25] for Gaussian, [43] and [4] for Poisson).

Proposition 6.9. A Gaussian (resp. Poisson) self-joining of an ergodic Gaussian system (resp. Poisson suspension) is ergodic. 


\section{GAGs AND PAPs}

7.1. From Foias-Stratila to GAGs. Lemańczyk, Parreau and Thouvenot introduced in [25] a class of Gaussian dynamical systems called GAGs (a French acronym meaning "Gaussian systems with Gaussian self-joinings"), on which they have developed a beautiful theory. The proof of the existence of GAGs relies on a striking rigidity result by Foias and Stratila [7], according to which there are some spectral measures that, in ergodic systems, can only be realized by Gaussian processes (this result improved a previous work of Sinai [49]). Foias-Stratila result can be formulated in the following way (see also [2, p. 375]).

Theorem 7.1 (Foias-Stratila). Let $(\Omega, \mathscr{F}, \mathbb{P}, T)$ be an ergodic probability-preserving dynamical sytem. Assume that for some nonzero real-valued $\xi_{0} \in L^{2}(\mathbb{P})$, the spectral measure of the stationary process $\left(\xi_{0} \circ T^{n}\right)_{n \in \mathbb{Z}}$ is continuous and concentrated on $K \cup(-K)$, where $K$ is a Kronecker subset of $\mathbb{T}$. Then the process $\left(\xi_{0} \circ T^{n}\right)_{n \in \mathbb{Z}}$ is Gaussian.

The above result is used in [25] through the following argument (which was already stated by Thouvenot $[51,50]$ ). Let us consider a Gaussian-Kronecker system $\boldsymbol{X}_{\sigma}$ (which means that the spectral measure $\sigma$ satisfies the same assumptions as in Theorem 7.1). Let $\nu$ be a self-joining of $\boldsymbol{X}_{\sigma}$, and denote by $\xi^{\prime}$ and $\xi^{\prime \prime}$ the two copies of the original Gaussian process with spectral measure $\sigma$ in the probabilitypreserving dynamical system

$$
\left(\mathbb{R}^{\mathbb{Z}} \times \mathbb{R}^{\mathbb{Z}}, \mathscr{B}\left(\mathbb{R}^{\mathbb{Z}} \times \mathbb{R}^{\mathbb{Z}}\right), \nu, S \times S\right),
$$

(as in Definition 6.3). In $L^{2}(\nu)$, let $H^{\prime}$ and $H^{\prime \prime}$ be the real subspaces spanned respectively by $\xi^{\prime}$ and $\xi^{\prime \prime}$, and $H:=H^{\prime}+H^{\prime \prime}$. For any $h \in H$, the spectral measure of the stationary process $\left(h \circ(S \times S)^{n}\right)_{n \in \mathbb{Z}}$ is absolutely continuous with respect to $\sigma$. Hence, if we further assume that $\nu$ is ergodic, Theorem 7.1 implies that $H$ is a Gaussian subspace.

We formalize the above by the following definition and theorem.

Definition 7.2. The Gaussian system $\boldsymbol{X}_{\sigma}$ is said to be a GAG when any ergodic self-joining of $\boldsymbol{X}_{\sigma}$ is a Gaussian self-joining.

Note that in this case, the system given by any ergodic self-joining is a generalized Gaussian system.

Theorem 7.3. Any Gaussian-Kronecker system is a GAG.

Even if Foias-Stratila result is the keystone for the construction of GAGs, it is shown in [25] that the class of GAGs extends far beyond the Gaussian-Kronecker systems. Indeed, it is proved that any simple-spectrum Gaussian system is GAG, and in particular there exist mixing GAGs.

Because of their special properties (see Section 7.3), GAGs can be used to produce very interesting examples of probability-preserving dynamical systems. But beside the theory of GAGs, Foias-Stratila property can lead to other surprising results. For example, it is used in [47] to show the existence of some spectral measures on the torus that can never be obtained in a transformation induced by an irrational rotation of the circle. 
7.2. Poissonian analog of Foias-Stratila Theorem and PAPs. Roughly speaking, an analog of Foias-Stratila Theorem in the context of Poisson suspensions should identify a special class of infinite measure-preserving dynamical systems $(X, \mathscr{A}, \mu, T)$-playing a role similar to the Kronecker measures--, such that any point process invariant and ergodic under the dynamics of $T$ would have a Poissonian character.

This invariance requirement is encompassed in the following notion of $T$-point process.

Definition 7.4. Let $(X, \mathscr{A}, \mu, T)$ be a sigma-finite measure-preserving system, and let $(\Omega, \mathbb{F}, \mathbb{P}, S)$ be a probability-preserving system. A point process $N: \Omega \rightarrow X^{*}$ is said to be a $T$-point process if

- for any $A \in \mathscr{A}: N(A) \circ S=N\left(T^{-1} A\right)$

- $N(A)=0 \mathbb{P}$-a.s. if $\mu(A)=0$.

The T-point process $N$ is $T$-free if its distribution is concentrated on the set of simple counting measures of the form $\sum_{i \in I} \delta_{x_{i}}$ such that for each $i \neq j, x_{i}$ and $x_{j}$ are not in the same T-orbit.

We assume now that $X$ is a complete and separable metric space, and that $\mu$ is an infinite and boundedly finite measure on $(X, \mathscr{A})$, that is a measure giving finite mass to any bounded measurable set. (This definition depends on the choice of the metric, and our assumption implies in particular that the metric is unbounded.)

We consider the family (FS) of $\mu$-preserving invertible transformations $T$ of $X$ satisfying:

- for each $n \geq 1,\left(X^{n}, \mathscr{A}^{\otimes n}, \mu^{\otimes n}, T^{\times n}\right)$ is ergodic ( $T$ is said to have infinite ergodic index);

- for each $n \geq 1$, if $\nu$ is a boundedly finite $T^{\times n}$-invariant measure on $\left(X^{n}, \mathscr{A}^{n}\right)$ whose marginals are absolutely continuous with respect to $\mu$, then $\left(X^{n}, \mathscr{A}^{\otimes n}, \nu, T^{\times n}\right)$ is conservative and any of its ergodic component $m$ is of the form

$$
m\left(A_{1} \times \cdots \times A_{n}\right)=\prod_{P \in \pi} \mu\left(\bigcap_{i \in P} T^{-k_{i}} A_{i}\right),
$$

where $\pi$ is a partition of $\{1, \ldots, n\}$ and $\left\{k_{i}\right\}$ are integers.

Observe that such an ergodic component gives rise to a system isomorphic to $\left(X^{\# \pi}, \mathscr{A}^{\otimes \# \pi}, \mu^{\otimes \# \pi}, T^{\times \# \pi}\right)$.

This above property is reminiscent to the concept of minimal self-joinings in the probability-preserving case. However we stress that, when the measure is infinite, greater care is required when dealing with invariant measures on Cartesian products (see [20] where this definition is discussed, and the construction of an example of transformation in the family (FS) is given).

We can now state the Poissonian version of Theorem 7.1, proved in [21].

Theorem 7.5 (Poissonian analog of Foias-Stratila Theorem). Let $N$ be a T-point process with moments of all orders defined on the ergodic probability-preserving dynamical sytem $(\Omega, \mathscr{F}, \mathbb{P}, S)$. If $T$ is in the family (FS) and if $N$ is $T$-free, then $N$ is a Poisson point process of intensity $\alpha \mu$ for some $\alpha>0$.

By analogy with GAG, we can formulate the following definition.

Definition 7.6. The Poisson suspension $\left(X^{*}, \mathscr{A}^{*}, \mu^{*}, T_{*}\right)$ is said to be a PAP when all its ergodic self-joinings are Poisson self-joinings. 
The analog of Theorem 7.3 in the context of Poisson suspensions has been established in [21].

Theorem 7.7. If $T$ is in the family (FS), then $\left(X^{*}, \mathscr{A}^{*}, \mu^{*}, T_{*}\right)$ is a PAP.

7.3. Properties of GAGs and PAPs. GAGs and PAPs have the great advantage that we can quite well describe the structure of their self-joinings. Since these self-joinings can tell a lot on the factors and centralizer of the corresponding transformation, it is no surprise that we are able to control the factors and centralizer of GAGs and PAPs.

We have seen in Section 6.1 that in the centralizer of any Gaussian system we can find transformations isomorphic to any other Gaussian system. It turns out that for GAGs, there is no other element in the centralizer.

Theorem 7.8 (Centralizer of a GAG [25]). Let $\boldsymbol{X}_{\sigma}$ be a GAG, and let $T \in$ $C(S)$. Then for each $\zeta$ in the real Gaussian subspace $H_{1}^{r}$ of $L^{2}\left(\mu_{\sigma}\right)$ spanned by the generating Gaussian process, we have $\zeta \circ T \in H_{1}^{r}$. In particular the system $\left(\mathbb{R}^{\mathbb{Z}}, \mathscr{B}\left(\mathbb{R}^{\mathbb{Z}}\right), \mu_{\sigma}, T\right)$ is a generalized Gaussian system.

We also identified in Section 6.1 a family of factors appearing in any standard Gaussian system. Again, in the case of GAGs, there is no other possible factor.

Theorem 7.9 (Factors of a GAG [25]). Let $\boldsymbol{X}_{\sigma}$ be a GAG. Any factor of $\boldsymbol{X}_{\sigma}$ is a classical factor. Any factor over which $S$ is relatively weakly mixing is a Gaussian factor.

The above theorem is used in [15]: in the case of a GAG system, the factor generated by a function of finitely many coordinates of the Gaussian process can only be the whole system or the even factor (and the latter occurs only when the function is even).

Here are the corresponding results for Poisson suspensions.

Theorem 7.10 (Centralizer of a PAP [21]). Let $\left(X^{*}, \mathscr{A}^{*}, \mu^{*}, T_{*}\right)$ be a PAP, and let $R \in C\left(T_{*}\right)$. Then there exists $S \in C(T)$ such that $R=S_{*}$.

In particular, when $T$ is in the family (FS), $C(T)$ is reduced to the powers of $T$ [16], thus $C\left(T_{*}\right)=\left\{T_{*}^{n}: n \in \mathbb{Z}\right\}$.

Theorem 7.11 (Factors of a PAP [21]). Any non trivial factor of the PAP $\left(X^{*}, \mathscr{A}^{*}, \mu^{*}, T_{*}\right)$ contains a non trivial Poisson factor. Any factor over which $T_{*}$ is relatively weakly mixing is a Poisson factor.

In particular, when $T$ is in the family (FS), $T_{*}$ has no non-trivial factor, as $T$ itself has no non-trivial factor [16]. Note that the primeness and the triviality of the centralizer of $T_{*}$ in this case are striking differences compared to Gaussian systems, which always possess a lot of factors and a large centralizer. The dissemblance can be pushed even further as it is proved (see [21] and [19]) that if $T$ is in the family (FS), $T_{*}$ is in fact disjoint from any standard Gaussian system.

\section{Future Directions}

A lot of questions remain open concerning the intersections of the classes of Gaussian systems or Poisson suspensions with other families of dynamical systems. In positive entropy, the situation is quite clear concerning the Gaussian case, but 
as already mentioned in Section 5.2 it is not known whether we can build a Poisson suspension which is $\mathrm{K}$ but not Bernoulli. In the zero-entropy setting, some results have been obtained for Gaussian systems: some are loosely Bernoulli, some are not [46], and we know that they cannot be of finite rank [48]. Similar issues for Poisson suspensions have not been studied yet. A general problem that arises is the question of disjointness of these systems of probabilistic origin with other classes such as finite-rank systems. One can hope for a unified answer taking advantage of the common features shared by Gaussian systems and Poisson suspensions, and inspired by ideas such as those developed in [4] or [28], where it is proved that both Gaussian systems and Poisson suspensions are disjoint from the class of distally simple systems.

For Poisson suspensions, the entropy theory is much richer and the situation where all three notions of entropy of a sigma-finite measure-preserving system do not coincide remains rather mysterious. For example, is it true that the Poisson entropy always dominates the Krengel entropy of the system?

It is noteworthy to mention that Poisson suspensions can also be defined over non-singular transformations, provided some integrability condition is satisfied. This is a potentially rich area to explore. (There is an ongoing work on this topic by A. Danilenko, Z. Kosloff and the second author of the present paper.)

Finally, we point out that this presentation focused on $\mathbb{Z}$-actions, eventhough the notions of Gaussian systems and Poisson suspensions extend naturally to more general group actions. For flows ( $\mathbb{R}$-actions), Gaussian systems and Poisson suspensions provide interesting examples of flows for which the self-similarity set

$$
I(T):=\left\{s \in \mathbb{R}:\left(T^{s t}\right)_{t \in \mathbb{R}} \text { is isomorphic to }\left(T^{t}\right)_{t \in \mathbb{R}}\right\}
$$

can be fully described (see $[9,3,10]$ ). For other groups, the generalization of some topics presented in the present survey is not always obvious. The entropy of Gaussian actions of Abelian groups is proved to be 0 or $\infty$ in [26], however it is unknown if the same holds for countable amenable groups. We can also ask for which group actions we have a Foias-Stratila theory.

\section{REFERENCES}

1. S. Attal, Lectures in Quantum noise Theory, http://math.univ-lyon1.fr/ attal/chapters.html.

2. I.P. Cornfeld, S. V. Fomin, and Ya. G. Sinai, Ergodic theory, Grundlehren der Mathematischen Wissenschaften [Fundamental Principles of Mathematical Sciences], vol. 245, Springer-Verlag, New York, 1982.

3. Alexandre I. Danilenko and Valery V. Ryzhikov, On self-similarities of ergodic flows., Proc. Lond. Math. Soc. (3) 104 (2012), no. 3, 431-454 (English).

4. Y. Derriennic, K. Fraczek, M. Lemańczyk, and F. Parreau, Ergodic automorphisms whose weak closure of off-diagonal measures consists of ergodic self-joinings., Colloq. Math. 110 (2008), no. 1, 81-115 (English).

5. R. L. Dobrushin, On the Poisson law for the distribution of particles in space., Ukr. Mat. Zh. 8 (1956), 127-134 (Russian).

6. Joseph L. Doob, Stochastic processes., New York: Wiley. 654 S. (1953)., 1953 (English).

7. C. Foias and S. Stratila, Ensembles de Kronecker dans la Théorie ergodique, C. R. Acad. Sci. Paris 267 (1967), 166-168.

8. S. Fomin, On dynamical systems in a space of functions., Ukr. Mat. Zh. 2 (1950), no. 2, 25-47 (Russian).

9. K. Fraczek and M. Lemańczyk, On the self-similarity problem for ergodic flows., Proc. Lond. Math. Soc. (3) 99 (2009), no. 3, 658-696 (English).

10. Krzysztof Fraczek, Joanna Kułaga, and Mariusz Lemańczyk, On the self-similarity problem for Gaussian-Kronecker flows., Proc. Am. Math. Soc. 141 (2013), no. 12, 4275-4291 (English). 
11. I. V. Girsanov, Spectra of dynamical systems generated by stationary Gaussian processes., Dokl. Akad. Nauk SSSR 119 (1958), 851-853 (Russian).

12. Sheldon Goldstein, Joel L. Lebowitz, and Michael Aizenman, Ergodic properties of infinite systems., Dyn. Syst., Theor. Appl., Battelle Seattle 1974 Renc., Lect. Notes Phys. 38, 112-143 (1975)., 1975.

13. Guillermo Grabinsky, Poisson process over $\sigma$-finite Markov chains., Pac. J. Math. 111 (1984), 301-315 (English).

14. Kiyosi Ito, On the ergodicity of a certain stationary process., Proc. Imp. Acad. Tokyo 20 (1944), 54-55 (English).

15. A. Iwanik, M. Lemańczyk, T. de la Rue, and J. de Sam Lazaro, Quelques remarques sur les facteurs des systèmes dynamiques gaussiens., Stud. Math. 125 (1997), no. 3, 247-254 (French).

16. É. JANVREsse, E. Roy, and T. DE LA RuE, Invariant measures for Cartesian powers of Chacon infinite transformation, Israel Journal of Mathematics 224 (2018), 1-37.

17. Élise Janvresse and Thierry De La Rue, Zero Krengel entropy does not kill Poisson entropy., Ann. Inst. Henri Poincaré, Probab. Stat. 48 (2012), no. 2, 368-376 (English).

18. Élise Janvresse, Tom Meyerovitch, Emmanuel Roy, and Thierry de la Rue, Poisson suspensions and entropy for infinite transformations., Trans. Am. Math. Soc. 362 (2010), no. 6, 3069-3094 (English).

19. É. Janvresse, E. Roy, and T. de la Rue, Ergodic poisson splittings, preprint.

20. N Nearly finite Chacon transformation, hal-01586869, 2017.

21. _ Poisson suspensions and SuShis, Ann. Scient. Éc. Norm. Sup. 50 (2017), no. 6, 1301-1334.

22. Steven Kalikow, A Poisson random walk is Bernoulli., Commun. Math. Phys. 81 (1981), 495-499 (English).

23. Ulrich Krengel, Entropy of conservative transformations, Z. Wahrscheinlichkeitstheorie und Verw. Gebiete 7 (1967), 161-181. MR MR0218522 (36 \#1608)

24. Günter Last and Mathew Penrose, Lectures on the Poisson process., vol. 7, Cambridge: Cambridge University Press, 2018 (English).

25. M. Lemańczyk, F. Parreau, and J.-P. Thouvenot, Gaussian automorphisms whose ergodic self-joinings are Gaussian, Fund. Math. 164 (2000), no. 3, 253-293.

26. Mariusz Lemánczyk, Entropy of Gaussian actions for countable Abelian groups., Fundam. Math. 157 (1998), no. 2-3, 277-286 (English).

27. Mariusz Lemańczyk, Spectral theory of dynamical systems, pp. 1618-1638, Springer New York, New York, NY, 2011.

28. Mariusz Lemańczyk, François Parreau, and Emmanuel Roy, Joining primeness and disjointness from infinitely divisible systems., Proc. Am. Math. Soc. 139 (2011), no. 1, 185-199 (English).

29. V. P. Leonov, The use of the characteristic functional and semi-invariants in the ergodic theory of stationary processes., Sov. Math., Dokl. 1 (1960), 878-881 (English).

30. F.A. Marchat, Ph.D. thesis, 1978.

31. G. Maruyama, The harmonic analysis of stationary stochastic processes., Mem. Fac. Sci. Kyūsyū Univ., Ser. A 4, 45-106 (1949), 1949

32. _ A singular flow with countable Lebesgue spectrum., J. Math. Soc. Japan 19 (1967), 359-365 (English).

33. _ Infinitely divisible processes, Theory Probab. Appl. 15 (1970), no. 1, 1-22.

34. D. Menchoff, Sur l'unicité du développement trigonométrique., C. R. Acad. Sci., Paris 163 (1916), 433-436 (French).

35. Yu. A. Neretin, Categories of symmetries and infinite-dimensional groups. Transl. from the Russian by G. G. Gould., Oxford: Clarendon Press, 1996 (English).

36. D. Newton, On Gaussian processes with simple spectrum., Z. Wahrscheinlichkeitstheor. Verw. Geb. 5 (1966), 207-209 (English).

37. Coalescence and spectrum of automorphisms of a Lebesgue space., Z. Wahrscheinlichkeitstheor. Verw. Geb. 19 (1971), 117-122 (English).

38. D. Newton and William Parry, On a factor automorphism of a normal dynamical system., Ann. Math. Stat. 37 (1966), 1528-1533 (English).

39. Donald S. Ornstein, Ergodic theory, randomness, and dynamical systems., Yale University Press, 1974 (English). 
40. William Parry, Entropy and generators in ergodic theory, W. A. Benjamin, Inc., New YorkAmsterdam, 1969. MR MR0262464 (41 \#7071)

41. M. S. Pinsker, Dynamical systems with completely positive or zero entropy., Sov. Math., Dokl. 1 (1960), 937-938 (English).

42. E. Roy, Poisson suspensions and infinite ergodic theory, Ergodic Theory Dynam. Systems 29 (2009), no. 2, 667-683.

43. Emmanuel Roy, Mesures de poisson, infinie divisibilité et propriétés ergodiques, Ph.D. thesis, 2005.

44. Thierry de la Rue, Entropie d'un système dynamique gaussien: cas d'une action de $\mathbb{Z}^{d}$., C. R. Acad. Sci., Paris, Sér. I 317 (1993), no. 2, 191-194 (French).

45. - Mouvement moyen et système dynamique gaussien., Probab. Theory Relat. Fields 102 (1995), no. 1, 45-56 (French).

46. - Systèmes dynamiques gaussiens d'entropie nulle, lâchement et non lâchement Bernoulli., Ergodic Theory Dyn. Syst. 16 (1996), no. 2, 379-404 (French).

47. L'induction ne donne pas toutes les mesures spectrales., Ergodic Theory Dyn. Syst. 18 (1998), no. 6, 1447-1466 (French).

48. _ Rang des systèmes dynamiques gaussiens., Isr. J. Math. 104 (1998), 261-283 (French).

49. Ya. G. Sinai, On higher order spectral measures of ergodic stationary processes., Theory Probab. Appl. 8 (1963), 429-436 (English).

50. J.-P. Thouvenot, Utilisation des processus gaussiens en théorie ergodique., Hommage à P. A. Meyer et J. Neveu, Paris: Société Mathématique de France, 1996, pp. 303-308 (French).

51. Jean-Paul Thouvenot, The metrical structure of some Gaussian processes., Ergodic theory and related topics II, Proc. Conf., Georgenthal/GDR 1986, Teubner-Texte Math. 94, 195-198 (1987)., 1987.

52. Haruo Totoki, The mixing property of Gaussian flows., Mem. Fac. Sci., Kyushu Univ., Ser. A 18 (1964), 136-139 (English).

53. A. M. Vershik, I. M. Gel'fand, and M. I. Graev, Representations of the group of diffeomorphisms., Russ. Math. Surv. 30 (1975), no. 6, 1-50 (English).

Élise Janvresse: Laboratoire Amiénois de Mathématique Fondamentale et Appliquée, CNRS-UmR 7352, Université de Picardie Jules Verne, 33 rue Saint Leu, F80039 Amiens CEDEX 1, FRANCE.

E-mail address: Elise.Janvresse@u-picardie.fr

Emmanuel Roy: Laboratoire Analyse, Géométrie et Applications, Université Paris 13 Institut Galilée, 99 avenue Jean-Baptiste Clément F93430 Villetaneuse, France.

E-mail address: roy@math.univ-paris13.fr

Thierry de la Rue: Laboratoire de Mathématiques Raphä̈l Salem, Université de Rouen, CnRS, Avenue de l'Université, F76801 Saint Étienne du Rouvray, France.

E-mail address: Thierry.de-la-Rue@univ-rouen.fr 